\title{
Determination of Copper in Water, Vegetables, Foodstuffs and Pharmaceuticals by Direct and Derivative Spectrophotometry
}

\author{
K. ASHOK RAO*, S. SIVARAMAIAH*, K. SARA SUBUDHI, \\ D. SREEVANI, Y. UMAMAHESWARI and G. V. HARITHA
}

Research Laboratories, Department of Chemistry, V. R. Group of Institutions Nellore, A.P. India

ashokrao.research@gmail.com

Received 7 June 2012 /Accepted 22 June 2012

\begin{abstract}
The quantification of copper in water, vegetables, foodstuffs, human hair and pharmaceutical samples was determined by a simple more sensitive and selective spectrophotometric

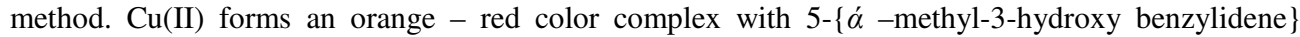
rhodanine [5M, 3H-BR], at pH 5.5 in sodium acetate and acetic acid buffer. The maximum absorbance was measured at $430 \mathrm{~nm}$. The Beer's law is obeyed in the range of $(0.05 \mu \mathrm{g}-13 \mu \mathrm{g} / \mathrm{mL})$. The molar absorptivity $(\varepsilon)$ and the Sandell's sensitivity of the complex were $0.6027 \times 10^{4} \mathrm{~mol}^{-1} \mathrm{~cm}^{-1}$ and $0.01054 \mu \mathrm{g} \mathrm{cm}^{-2}$ respectively. First, second and third derivative spectrophotometry were also proposed and employed successfully for the determination of copper in the supra. The performance of the present method was also evaluated in terms of RMSEP, REP and RSD, students $t$ - test. This indicates the greater importance of the method than other methods reported in the literature.
\end{abstract}

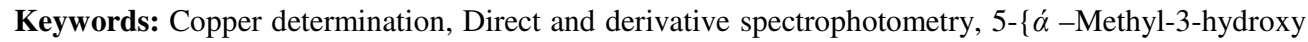
benzylidene\} rhodanine, RMSEP, REP, RSD

\section{Introduction}

Metals at trace levels are components of natural biosphere. Hence they are required for body structure, fluid balance, protein and to produce hormones. Some of them are considered essential, but at high concentration they are toxic. The range between essentiality and toxicity is often very small. Copper occurs in nature as mineral compounds, $75 \%$ copper that is mined is used in the electrical industries, house hold-utensil, metallic blends and pigments ${ }^{1}$. From these sources, it will enter as pollutant and pollutes the water, soil, foodstuffs, flora and fauna.

It is an essential to activate enzymes involved in $^{2}$ cellular respiration- (cytochrome-C oxidase), iron oxidation- (ceruloplasmin), connective tissue formation-(lysyl oxidase) neuro transmitter biosynthesis-(Mono Amine oxidase) and pigment formation-(Tyrosanase). On over healthy limit accumulates in the liver causing dizziness, vomiting, diarrhoea, transpiration and depending on its concentration it leads to death ${ }^{3-7}$. In chronic exposure, liver, kidney and spleen may be injured and may develop anemia. The deficiency of copper causes the coronary, 
artery; heart diseases and can promote connection between sugar molecules and protein molecules which results in tissue damification in diabetic people $\mathrm{e}^{9-10}$. Therefore, from this point of view, it is necessary to establish a rapid simple, sensitive and accurate procedure for the determination of copper concentration. Several techniques have been used for the determination of copper in different samples ${ }^{11-15}$. However these methods have the disadvantages that the operation of the instrumentation used, is complex and the price of the instrumentation is expensive compared with UV-visible spectrophotometry.

Hitherto several complexing agents ${ }^{16-25}$ are reported for the spectrophotometric determination of copper. Spectrophotometry still represents an attractive technique for the determination of metal ions in aqueous media, because of its simplicity, being inexpensive and is readily availabble ${ }^{26}$. Therefore in the present investigation a selective reagent $5-\{\dot{\alpha}-$ methyl-3-hydroxy benzylidene \}rhodanine [5M,3H-BR] was chosen for the UV- visible spectrophotometric determination of copper(II) in the samples selected.

\section{Experimental}

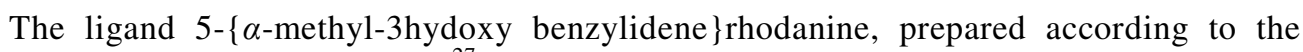
procedure reported previously ${ }^{27} .120 \mathrm{mg}$ of ammonium acetate was added to a mixture of $360 \mathrm{~mL}$ glacial acetic acid and $13 \mathrm{~mL}$ benzene then $2 \mathrm{~g}$ of rhodanine was added. The reaction mixture was stirred and boiled for 5 minutes. $2 \mathrm{~g}$ of 3 -hydroxy acetophenone was then added to the reaction mixture then refluxed to overnight. Later it was allowed to cool at room temperature which gives a yellow precipitate. It is separated by filtration, washed with water and purified by recrystallization from methanol/ water (1:1) mixture melting point is $201{ }^{0} \mathrm{C}-202{ }^{\circ} \mathrm{C}$. The structure was confirmed from Mass IR, NMR spectra.

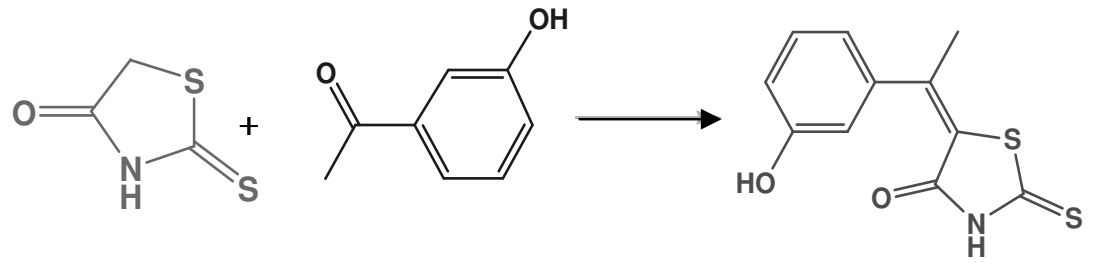

Scheme 1. Formation of 5-\{ $\dot{\alpha}$-methyl-3-hydroxy benzylidene $\}$ rhodanine

\section{Preparation of solutions}

All the chemicals were of AnalaR grades from Fisher Scientific Qualigens, India.

$\mathrm{Cu}(\mathrm{II})$ - solution

Stock standard $\mathrm{Cu}(\mathrm{II})$ solution was prepared by dissolving $0.3929 \mathrm{~g}$ of $\mathrm{Cu}(\mathrm{II})$ sulphate pentahydrte in double distilled water containing $1000 \mu \mathrm{g} / \mathrm{mL}$. The solution was standardized by idometry ${ }^{28}$. The working standard solutions were prepared by suitable dilution of the stock solution.

\section{Buffer solutions}

Buffer solutions were prepared by employing $0.1 \mathrm{M}$ acetic acid and $0.1 \mathrm{M}$ sodium acetate s9-30 $^{20}$ in the $\mathrm{pH}$ range 3-10. Borate buffers were also prepared in the $\mathrm{pH}$ range 3-12 from $1 \mathrm{M}$ boric acid adjusting with $1 \mathrm{M}$ sodium hydroxide. 


\section{Solutions of diverse ions}

Solutions of diverse ions containing $1000 \mu \mathrm{g} / \mathrm{mL}$ were prepared by dissolving required amounts of salts of the corresponding ions in double distilled water

\section{Reagent solution}

The reagent stock solution $(0.1 \mathrm{M})$ was prepared by dissolving $1.255 \mathrm{~g}$ of $[5 \mathrm{M}, 3 \mathrm{H}, \mathrm{BR}]$ in DMF or methanol. This was diluted to the required concentration using $40 \% \mathrm{DMF}$.

\section{Instruments}

Elico micro processor based double beam UV - visible spectrophotometer SL 210 equipped with $1 \mathrm{~cm}$ quartz cells were used for spectrophotometric measurements. The $\mathrm{pH}$ measurements are made with Elico digital pH meter L.I 127 model.

\section{General procedure for studies of different parameters}

To ensure the complexation ratio between the $\mathrm{Cu}(\mathrm{II})$ and $[5 \mathrm{M}, 3 \mathrm{H}-\mathrm{BR}]$, and to quantification of the $\mathrm{Cu}(\mathrm{II})$ in the sample solutions the following procedure was performed. To an aliquots of sample solution containing $\mu \mathrm{g}$ quantities of $\mathrm{Cu}$ (II) was added to a series of comparison tubes followed by $5 \mathrm{~mL}$ of acetic acid and sodium acetate buffer to adjust the pH5.5, then equilibrated with $5 \mathrm{~mL}$ of [ $5 \mathrm{M}, 3 \mathrm{H}-\mathrm{BR}$ ] solution (in $40 \% \mathrm{DMF}$ ) for $10 \mathrm{~min}$ and diluted to $20 \mathrm{~mL}$ with double distilled water. The absorbance of orange - red color complex formed was measured against a similarly prepared reagent blank at $430 \mathrm{~nm}$. The composition of the complex was computed by Job's continuous variation, mole ratio and slope ratio methods. The amount of $\mathrm{Cu}$ (II) present in the sample solutions ${ }^{31-38}$ prepared, were computed from the standard calibration carves in the range 0.05 to $13 \mu \mathrm{g}$ both by inspecting the direct and derivative spectra.

\section{Results and Discussion}

The absorption spectra of an orange-red color complex of [Cu(II) - 5M, 3H, BR] were recorded in the wave length region $400-600 \mathrm{~nm}$ against the reagent blank (Figure 1). It was observed that complex showed the maximum absorbance at $420 \mathrm{~nm}$ in borate buffers and at $430 \mathrm{~nm}$ in acetate buffers, whereas in acetate buffers the complex absorbance was found to be maximum. Hence, the $430 \mathrm{~nm}$ in acetate buffers were chosen for the proposed studies

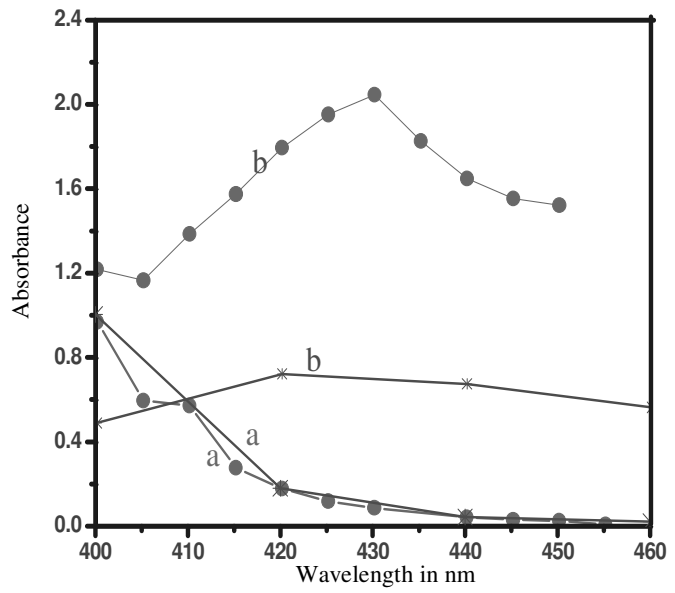

Figure 1. Absorption spectra of; a) $5 \mathrm{M} 3 \mathrm{H}$ BR vs. buffer blank; b) $\mathrm{Cu}(\mathrm{II})-5 \mathrm{M} 3 \mathrm{H}$ BR complex vs. reagent blank acetate buffer ${ }^{*}$ Borate buffer $\mathrm{Cu}(\mathrm{II})=1.6 \times 10^{-3} \mathrm{M}(100 \mu \mathrm{g}), 5 \mathrm{M}$ $3 \mathrm{H} \mathrm{BR}=3 \times 10^{-3} \mathrm{M}$ 


\section{Effect of the $\mathrm{pH}$}

The $\mathrm{pH}$ of the aqueous solution is an important parameter for complex formation. The influence of $\mathrm{pH}$ of the aqueous solutions on the formation of [Cu(II) - 5M, 3H, BR] complex were investigated at $430 \mathrm{~nm}$ using various buffer solutions of different $\mathrm{pH}$ values (Figure 2). The complex with maximum absorbance was observed at $\mathrm{pH}$ values 5 to 8 in acetate buffers and 8 to 10 in borate buffers. However, the maximum absorbance was found in acetate buffers. In the light of these findings all subsequent studies were carried out at $\mathrm{pH} 5.5$ for direct and derivative spectrophotometry
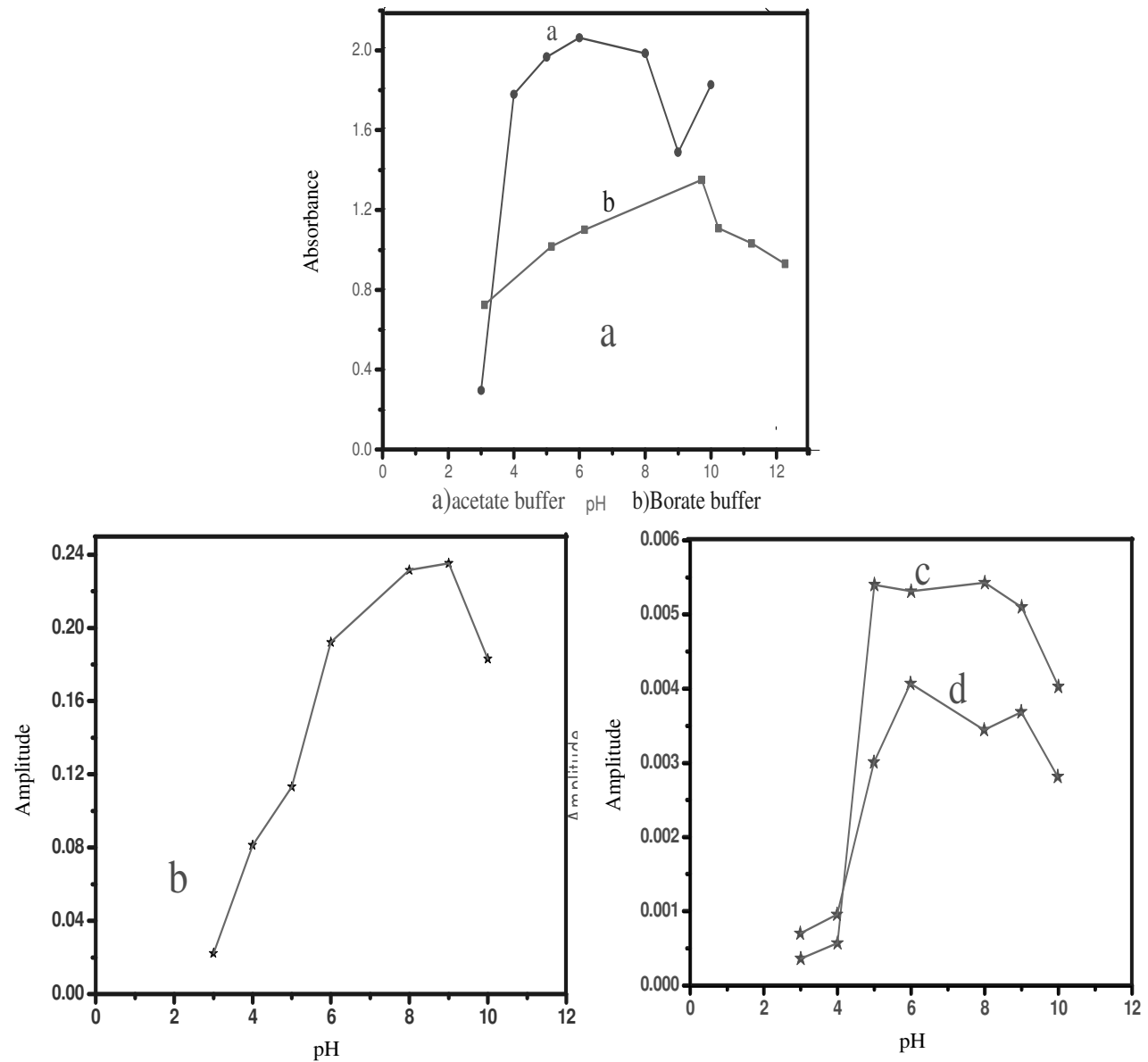

Figure 2. Effect of $\mathrm{pH}$ on the absorbance of $[\mathrm{Cu}(\mathrm{II})-5 \mathrm{M} 3 \mathrm{H}$ BR] system (a) Direct spectrophotometry (b) $1^{\text {st }}$ derivative (c) $2^{\text {nd }}$ derivative (d) $3^{\text {rd }}$ derivative $\mathrm{Cu}(\mathrm{II})=[5 \mathrm{M} 3 \mathrm{H}$ BR] $=3 \times 10^{-4} \mathrm{M}$

\section{Effect of solvent and reagent concentration}

A tenfold molar excess of the reagent was necessary for the maximum color development. An orange - red color formation between $\mathrm{Cu}$ (II) and reagent was instantaneous and the color was stable for more than 36 hours. The complex was found to soluble in $40 \%$ of DMF. So the reagent solutions were prepared in $40 \%(\mathrm{v} / \mathrm{v})$ DMF. 


\section{Effect of salting out agent}

The complexation of $\mathrm{Cu}(\mathrm{II})$ with the $(5 \mathrm{M}, 3 \mathrm{H}, \mathrm{BR})$ is certain and effective at $\mathrm{pH} 5.5$. However various salting out agents such as sodium sulphate, sodium chloride, ammonium chloride, ammonium sulphate and sodium carbonate are used for the enhancement of the color of the metal complex in the analysis of the different samples. It was observed that, the presence of $0.01 \mathrm{M}$ sodium corbonate effectively increases the color (Figure 3).

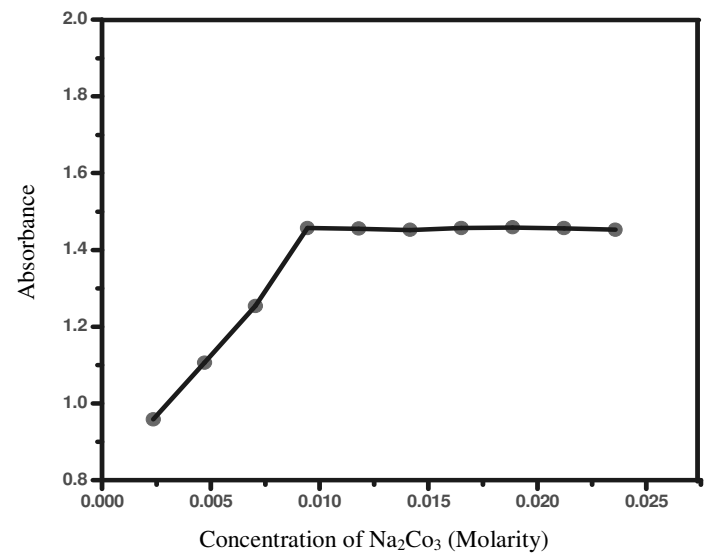

Figure 3. Effect of $\mathrm{Na}_{2} \mathrm{CO}_{3}$ on the complexation

\section{Nature of the complex}

The composition of the complex and stoichiometric ratio between the metal to ligand was determined by mole ratio, slope ratio and jobs continuous variation methods elating of these experimental results indicates the $\mathrm{Cu}(\mathrm{II})$ forming the 1:4 complex with the reagent and the stoichiometric ratio is 1:2. So the reagent was found to be a bidental ligand (Figure 4-6).

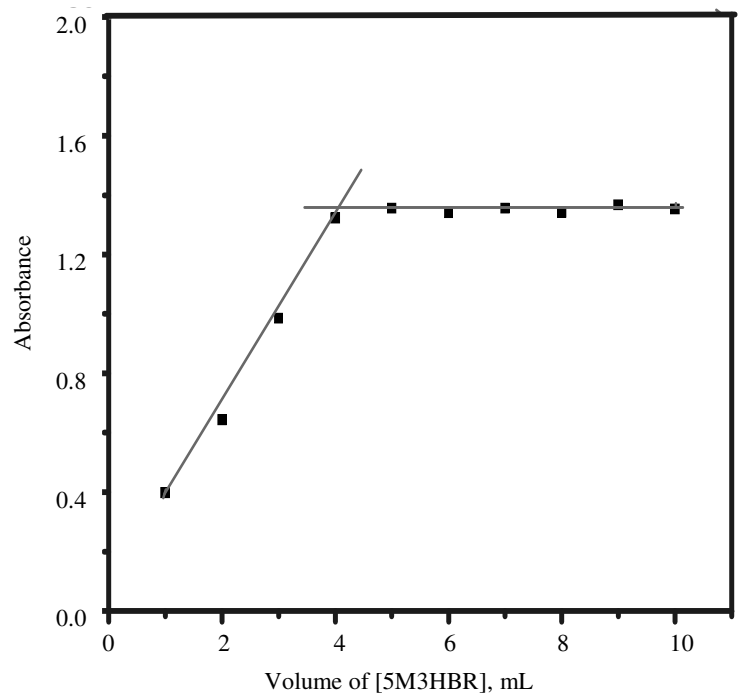

Figure 4. Mole ratio plot, $\mathrm{pH}: 5.5 \mathrm{Cu}(\mathrm{II})=[5 \mathrm{M} 3 \mathrm{H} \mathrm{BR}]=1.6 \times 10^{-4} \mathrm{M}$, volume of $\mathrm{Cu}(\mathrm{II})=1 \mathrm{~mL}$ $(10 \mu \mathrm{g})$ 


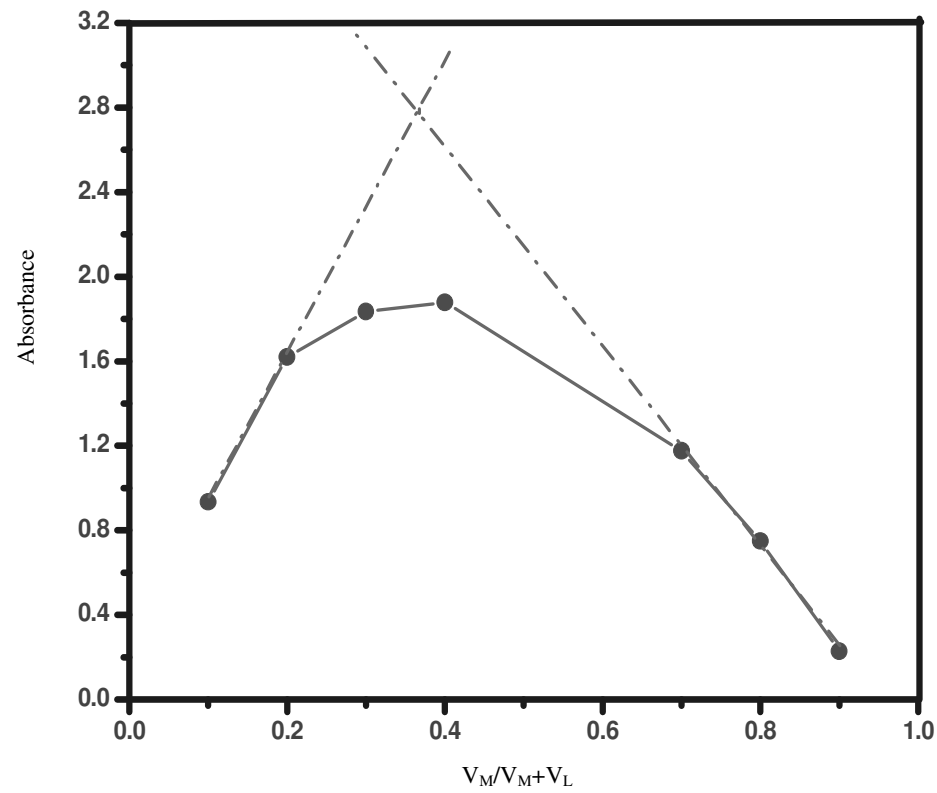

Figure 5. Job's continuous variation method for the $\mathrm{Cu}(\mathrm{II})-[5 \mathrm{M} 3 \mathrm{H} \mathrm{BR}]$ complex, $\mathrm{Cu}(\mathrm{II})=$ $[5 \mathrm{M} 3 \mathrm{H}$ BR $]=1.6 \times 10-4 \mathrm{M}, \mathrm{pH}: 5.5, \lambda_{\max }: 430 \mathrm{~nm}$
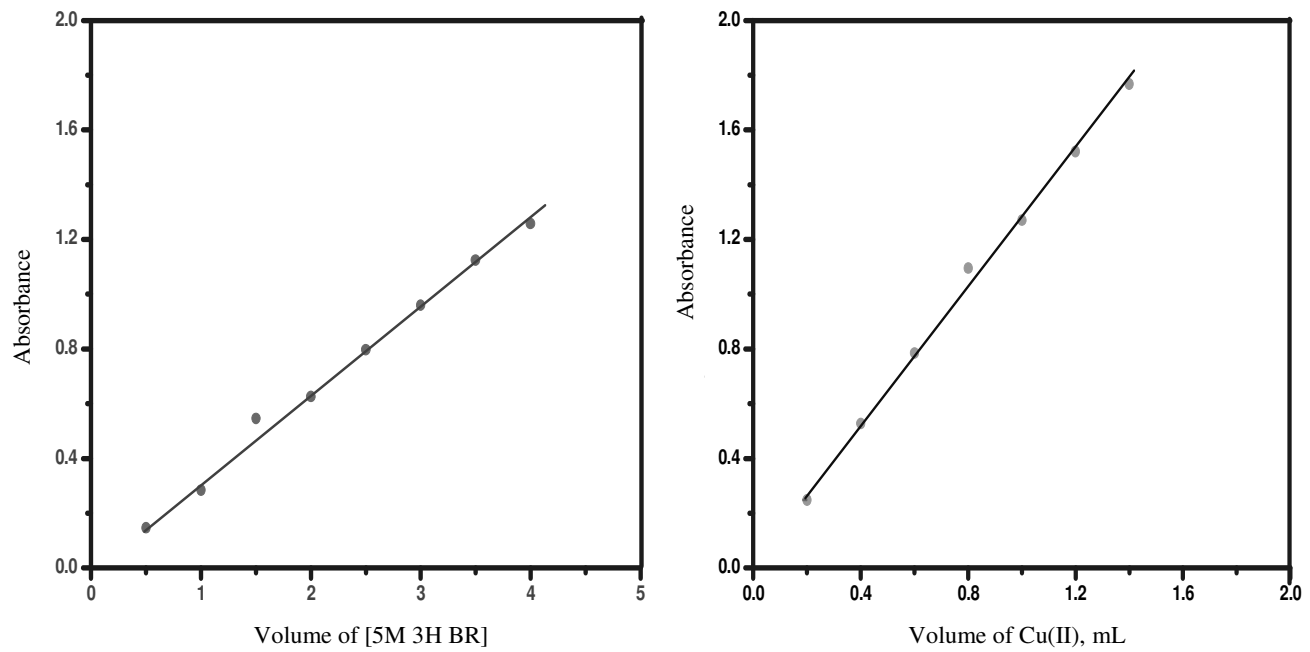

Figure 6. Slope ratio method, $\mathrm{Cu}(\mathrm{II})=[5 \mathrm{M} 3 \mathrm{H}$ BR $]=1.6 \times 10^{-4} \mathrm{M}$ pH:5.5 $\lambda_{\max }=430$

\section{Performance for the calibration of proposed method}

Beer's law was obeyed in the concentration range $0.05-13 \mu \mathrm{g} / \mathrm{mL}$ of $\mathrm{Cu}$ (II) in different sample solutions. The molar absorptivity of the complex was $0.6027 \times 10^{4} \mathrm{moles}^{-1} \mathrm{~cm}^{-1}$. The Sandell's sensitivity of the method was found to be $0.01054 \mu \mathrm{gcm}^{-2}$. The standard deviation, correlation coefficient and other statistical parameters of the method are evaluated to ten replicate determinations Table 1. 
Table 1. Performance data for the calibration of proposed method

\begin{tabular}{|c|c|c|c|c|c|c|}
\hline 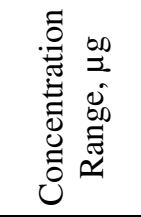 & 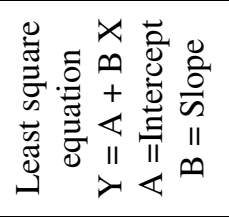 & 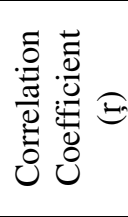 & 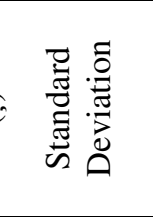 & $\hat{\mathscr{A}}_{0}$ & $\frac{0}{x}$ o & $\begin{array}{c}\text { Amount determined } \\
\text { In ten replicate samples, } \mu \mathrm{g}\end{array}$ \\
\hline $0.05-0.5$ & $\begin{array}{l}Y=-0.00207 \\
+0.2398 X\end{array}$ & 1.0000 & 0.000769 & 0.1917 & 0.2991 & $\begin{array}{c}0.4010,0.3997,0.4022,0.4012 \\
0.4020,0.4012,0.4018 \\
0.4014,0.4012,0.4024\end{array}$ \\
\hline $0.5-5.0$ & $\begin{array}{c}Y=-0.0364+ \\
0.2466 X\end{array}$ & 1.000 & 0.0505 & 1.2241 & 2.6839 & $\begin{array}{c}4.1625,4.1091,4.0275,4.1525 \\
4.1592,4.0761,4.1475,4.1855 \\
4.1572,4.0761 .\end{array}$ \\
\hline $5.0-13$ & $\begin{array}{l}\mathrm{Y}=1.4559+ \\
0.0103 \mathrm{X}\end{array}$ & 0.9869 & 0.005957 & 0.0799 & 0.2014 & $\begin{array}{c}\text { 7.4550,7.4469,7.4470,7.4481 } \\
7.4500,7.4470,7.4620 \\
7.4475,7.4451,7.4598 \\
\end{array}$ \\
\hline
\end{tabular}

\section{Derivative spectrophotometry}

For the determination of copper derivative spectrophotometric methods are also developed. The $1^{\text {st }}$ and $2^{\text {nd }}$ derivative spectra show the maximum amplitude at $405 \mathrm{~nm}$ and $430 \mathrm{~nm}$. The $3^{\text {rd }}$ derivative curve amplitude becomes zero at $428 \mathrm{~nm}$ and maxis mum amplitude was shifted to $435-460 \mathrm{~nm}$. Calibration plots drawn between the amplitude and the concentration of $\mathrm{Cu}(\mathrm{II})$ was found to be linear in the range $(0.05-13 \mu \mathrm{g} / \mathrm{mL})$ The derivative amplitudes were found to be proportional to the concentration of $\mathrm{Cu}$ (II). The results are summarized in Table 2, Figure 7.

Table 2. Calibration data for the derivative spectrophotometric determination

\begin{tabular}{|c|c|c|c|}
\hline $\begin{array}{l}\text { Linear Range, } \\
\mu \mathrm{g} / \mathrm{mL}\end{array}$ & $\begin{array}{c}\text { Calibration Equation } \\
\mathrm{Y}=\mathrm{A}+\mathrm{BX} \\
\mathrm{A}=\text { Intercept }, \mathrm{B}=\text { Slope }\end{array}$ & $\begin{array}{l}\text { Wavelength, } \\
\mathrm{nm}\end{array}$ & $\begin{array}{l}\text { Correlation } \\
\text { Coefficient (rg) }\end{array}$ \\
\hline \multirow{3}{*}{$0.05-0.5$} & $\begin{array}{c}\text { First }- \text { Derivative Spectrophotometry } \\
\partial \mathrm{A} / \partial \lambda=-0.0292+0.564 \mathrm{X}\end{array}$ & 405 & 0.9985 \\
\hline & $\begin{array}{c}\text { Second - DerivativeSpectrophotometry } \\
\partial^{2} \mathrm{~A} / \partial \lambda^{2}=-0.0089+0.0440 \mathrm{X}\end{array}$ & 430 & 1.003 \\
\hline & $\begin{array}{c}\text { Third - Derivative Spectrophotometry } \\
\partial^{3} \mathrm{~A} / \partial \lambda^{3}=-0.000124+0.0046 \mathrm{X}\end{array}$ & $435-460$ & 0.9989 \\
\hline \multirow{3}{*}{$0.5-5.0$} & $\begin{array}{c}\text { First }- \text { Derivative Spectrophotometry } \\
\partial \mathrm{A} / \partial \lambda=-0.0431+0.0588 \mathrm{X}\end{array}$ & 405 & 0.9937 \\
\hline & $\begin{array}{c}\text { Second - DerivativeSpectrophotometry } \\
\partial^{2} \mathrm{~A} / \partial \lambda^{2}=-0.000526+0.001207 \mathrm{X}\end{array}$ & 430 & 0.9980 \\
\hline & $\begin{array}{l}\text { Third - Derivative Spectrophotometry } \\
\partial^{3} \mathrm{~A} / \partial \lambda^{3}=-0.000116+0.0002656 \mathrm{X}\end{array}$ & $435-460$ & 1.000 \\
\hline \multirow{3}{*}{$5.0-13$} & $\begin{array}{c}\text { First }- \text { Derivative Spectrophotometry } \\
\partial \mathrm{A} / \partial \lambda=-0.0614+0.0534 \mathrm{X}\end{array}$ & 405 & 1.0032 \\
\hline & $\begin{array}{l}\text { Second - DerivativeSpectrophotometry } \\
\partial^{2} \mathrm{~A} / \partial \lambda^{2}=-0.008788+0.002201 \mathrm{X}\end{array}$ & 430 & 0.9887 \\
\hline & $\begin{array}{l}\text { Third - Derivative Spectrophotometry } \\
\partial^{3} \mathrm{~A} / \partial \lambda^{3}=-0.00223+0.00087 \mathrm{X}\end{array}$ & $435-460$ & 0.9907 \\
\hline
\end{tabular}



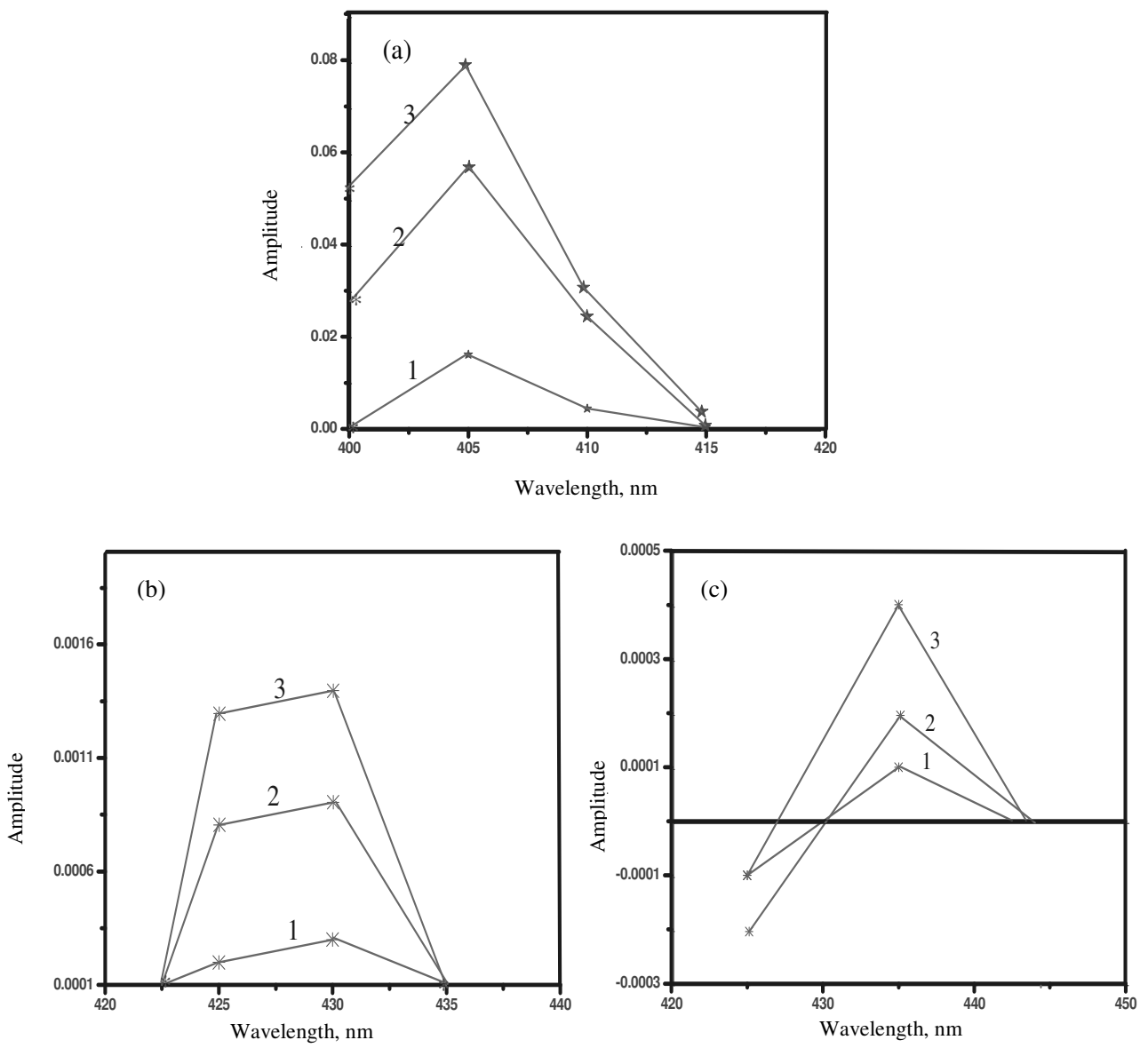

Figure 7. Derivative spectra of [Cu(II)-5M 3H BR] system (a) first order (b) second order (c) third order $\mathrm{Cu}(\mathrm{II})-\mu \mathrm{g} / \mathrm{mL}$ (1) 1.5 (2) 3 (3) 4.5

\section{Effect of diverse ions}

To examine the effect of the diverse ions, $10 \mu \mathrm{g}$ of $\mathrm{Cu}(\mathrm{II})$ and diverse ion in question were transferred in to comparison tubes of $20 \mathrm{~mL}$ capacity followed by an excess of reagent solution at $\mathrm{pH}$ 5.5. However, in the case of interference were masked using citrate, tartarate, phosphate as masking agents. The tolerance limit was stated as the highest amount for an ion that produces an error not exceeding $\pm 3 \%$ in the determination. The results are summarized in the Table 3 .

\section{Analytic conclusion}

The proposed direct and derivative spectrophotometric method were employed for the determination of $\mathrm{Cu}(\mathrm{II})$ in different samples such as natural water, biological samples, foodstuffs and pharmaceutical samples. The results are summarized in the Table $4,5 \& 6$. The WHO provisional guideline value of $2000 \mu \mathrm{g} / \mathrm{L}(2 \mu \mathrm{g} / \mathrm{mL})$ of copper in drinking water could produce an adverse reaction ${ }^{39}$. This is computable with the United states drinking water action level of $1300 \mu \mathrm{g} / \mathrm{mL}(1.3 \mu \mathrm{g} / \mathrm{mL})^{40}$. In the present method, the content of copper in buffalo's milk and Cow's milk were found to be $1065-1216 \mu \mathrm{g} / \mathrm{L}, 1230-1318 \mu \mathrm{g} / \mathrm{L}$ respectively. 
Table 3. Effect of diverse ions

\begin{tabular}{|c|c|c|}
\hline Diverse ion & Added as & Tolerance limit, $\mu \mathrm{g} / 20 \mathrm{~mL}$ \\
\hline $\mathrm{Mg}^{+2}$ & $\mathrm{MgSO}_{4}$ & 1000 \\
\hline $\mathrm{Ba}^{+2}$ & $\mathrm{BaCl}_{2}$ & 984 \\
\hline $\mathrm{Co}^{+2}$ & $\mathrm{Co}\left(\mathrm{NO}_{3}\right)_{2}$ & 675 \\
\hline $\mathrm{Ag}^{+}$ & $\mathrm{AgNO}_{3}$ & 750 \\
\hline $\mathrm{Pb}^{+2}$ & $\mathrm{~Pb}\left(\mathrm{NO}_{3}\right)_{2}$ & 688 \\
\hline $\mathrm{Se}^{+2}$ & $\mathrm{Na}_{2} \mathrm{SeO}_{3}$ & 943 \\
\hline $\mathrm{Ca}^{+2}$ & $\mathrm{CaCl}_{2}$ & 920 \\
\hline $\mathrm{Sn}^{+2}$ & $\mathrm{Sn}\left(\mathrm{NO}_{3}\right)_{2}$ & 1000 \\
\hline $\mathrm{Te}^{+2}$ & $\mathrm{Na}_{2} \mathrm{TeO}_{3}$ & 1000 \\
\hline $\mathrm{Li}^{+2}$ & $\mathrm{LiNO}_{3}$ & 785 \\
\hline $\mathrm{Al}^{+3}$ & $\mathrm{Al}\left(\mathrm{NO}_{3}\right)_{3}$ & 1034 \\
\hline $\mathrm{Cr}^{+3}$ & $\mathrm{~K}_{2} \mathrm{Cr}_{2} \mathrm{O}_{7}$ & 1000 \\
\hline $\mathrm{Zn}^{+2}$ & $\mathrm{ZnSO}_{4}$ & 902 \\
\hline $\mathrm{Cd}^{+2}$ & $\mathrm{CdCl}_{2}$ & 1220 \\
\hline $\mathrm{Hg}^{+2}$ & $\mathrm{HgCl}_{2}$ & 980 \\
\hline $\mathrm{Mn}^{+2}$ & $\mathrm{MnCl}_{2}$ & 650 \\
\hline $\mathrm{Ni}^{+2}$ & $\mathrm{NiSO}_{4}$ & 730 \\
\hline $\mathrm{Fe}^{+3}$ & $\mathrm{FeSO}_{4}$ & 945 \\
\hline
\end{tabular}

Table 4. Direct spectrophotometric determination

\begin{tabular}{|c|c|c|c|c|c|c|c|}
\hline Sample & $\begin{array}{c}\text { Amount of } \\
\text { Copper Spiked } \\
\mu \mathrm{g} / \mathrm{mL}\end{array}$ & $\begin{array}{l}\text { Amount of } \\
\text { copper found } \\
\mu \mathrm{g} / \mathrm{mL}\end{array}$ & $\begin{array}{c}\text { Recovery } \\
\%\end{array}$ & RMSEP & $\underset{\%}{\mathrm{REP}}$ & $\underset{\%}{\mathrm{RSD}}$ & $t$-test \\
\hline \multirow[t]{3}{*}{ Tap Water } & - & 0.0086 & - & 0.00033 & 9.696 & 3.820 & 3.3681 \\
\hline & 1.066 & $1.05 \pm 0.02$ & 97.7 & 0.0479 & 4.1862 & 4.562 & 0.8648 \\
\hline & 1.3351 & $1.333 \pm 0.01$ & 99.26 & 0.0101 & 1.7802 & 0.0757 & 0.7827 \\
\hline Pinakini & & 0.2408 & & 0.0182 & 3.8725 & 7.558 & 1.2336 \\
\hline \multirow[t]{2}{*}{ Water } & 0.76 & $0.982 \pm 0.03$ & 97.9 & 0.0109 & 2.0460 & 0.111 & 1.3054 \\
\hline & 0.824 & $1.051 \pm 0.05$ & 98.67 & 0.0283 & 5.7831 & 0.2693 & 1.4637 \\
\hline $\begin{array}{c}\text { Milk } \\
\text { (Buffalo) }\end{array}$ & - & 1.216 & - & 0.1460 & 1.3990 & 3.4312 & 0.3530 \\
\hline Milk(Cow) & - & 1.318 & - & 0.1657 & 4.9157 & 3.5917 & 0.1469 \\
\hline \multirow[t]{2}{*}{ Mother Milk } & - & 0.4841 & - & 0.0686 & 4.7901 & 2.0240 & 0.8205 \\
\hline & & $\mu \mathrm{g} / \mathrm{g}$ & & & & & \\
\hline \multirow[t]{2}{*}{ Cabbage } & - & 17.2 & - & 0.0779 & 1.2671 & 2.158 & 1.1731 \\
\hline & 2.0 & $18.6 \pm 0.001$ & 96.8 & 0.2319 & 4.6434 & 5.927 & 2.5153 \\
\hline Vegetable & - & 14.76 & - & 0.0642 & 0.8438 & 2.0742 & 1.6413 \\
\hline Banana & 8.0 & $21.98 \pm 0.006$ & 96.57 & 0.0563 & 0.4423 & 1.2197 & 2.2859 \\
\hline Amruthapani & - & 16.4 & - & 0.1254 & 6.4778 & 3.633 & 0.2370 \\
\hline Banana & 4.0 & $19.55 \pm 0.004$ & 95.8 & 0.1718 & 1.6557 & 0.3871 & 1.0878 \\
\hline \multirow[t]{2}{*}{ Tomato } & - & 18.35 & - & 0.2239 & 6.9181 & 5.782 & 17173 \\
\hline & 4.0 & $21.53 \pm 0.01$ & 96.33 & 0.4036 & 5.8121 & 5.500 & 1.6715 \\
\hline \multirow[t]{2}{*}{ Wheat flour } & - & 15.7 & - & 0.0506 & 3.1433 & 1.532 & 1.6810 \\
\hline & 5.0 & $20.4 \pm 0.03$ & 98.55 & 0.4040 & 4.1543 & 7.4252 & 0.0821 \\
\hline \multirow[t]{2}{*}{ Cucumber } & - & 24.28 & - & 0.0268 & 1.4955 & 0.5268 & 2.2065 \\
\hline & 4.0 & $27.11 \pm 0.001$ & 95.86 & 0.1526 & 4.8579 & 1.6495 & 2.4825 \\
\hline \multirow[t]{2}{*}{ Rice(1010) } & - & 14.522 & - & 0.2017 & 0.7729 & 3.9680 & 2.2105 \\
\hline & 5.0 & $17.7482 \pm 0.03$ & 90.91 & 0.7770 & 4.7914 & 12.508 & 1.8041 \\
\hline Rice & - & 21.68 & - & 0.1278 & 3.2275 & 2.8070 & 0.1855 \\
\hline (Masuria) & 3.0 & $23.93 \pm 0.0001$ & 96.96 & 0.1357 & 3.8526 & 2.6993 & 1.0136 \\
\hline Human hair & - & 41.2 & - & 0.0329 & 0.4409 & 0.38506 & 1.6724 \\
\hline
\end{tabular}


Table 5. Derivative spectrophotometric determination

\begin{tabular}{|c|c|c|c|c|c|c|c|}
\hline Sample & $\begin{array}{c}\text { Amount of } \\
\text { copper Spiked, } \\
\mu \mathrm{g} / \mathrm{mL}\end{array}$ & $\begin{array}{c}\text { Amount of } \\
\text { copper found } \\
\mu \mathrm{g} / \mathrm{mL}\end{array}$ & 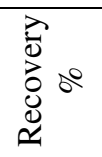 & 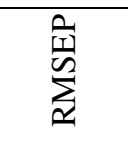 & $\begin{array}{l}\text { REP } \\
\%\end{array}$ & RSD & $t$ - test \\
\hline Tap Water & - & 0.0086 & - & 0.00033 & 9.696 & 3.820 & 3.3700 \\
\hline \multirow[t]{2}{*}{$1^{\text {st }}$ derivative } & 1.037 & $1.04 \pm 0.01$ & 99.4 & 0.0778 & 9.556 & 7.487 & 0.8454 \\
\hline & 1.60 & $1.59 \pm 0.04$ & 98.8 & 0.0283 & 5.720 & 1.780 & 5.7992 \\
\hline \multirow[t]{2}{*}{$2^{\text {nd }}$ derivative } & 0.896 & $0.87 \pm 0.06$ & 96.5 & 0.0424 & 2.607 & 4.857 & 1.3424 \\
\hline & 1.635 & $1.63 \pm 0.03$ & 99.4 & 0.0264 & 3.400 & 0.162 & 3.6413 \\
\hline \multirow[t]{2}{*}{$3^{\text {rd }}$ derivative } & 1.102 & $1.086 \pm 0.001$ & 97.8 & 0.0470 & 9.410 & 4.328 & 1.5676 \\
\hline & 0.792 & $0.781 \pm 0.07$ & 97.5 & 0.00712 & 2.929 & 0.913 & 0.9763 \\
\hline \multirow{2}{*}{$\begin{array}{l}\text { Pinakini } \\
\text { water }\end{array}$} & - & 0.2 & - & 0.0182 & 3.872 & 7.558 & 1.2336 \\
\hline & 0.781 & $1.022 \pm 0.03$ & 99.9 & 0.04127 & 3.359 & 4.040 & 2.1183 \\
\hline \multirow[t]{2}{*}{$1^{\text {st }}$ derivative } & 1.16 & $1.384 \pm 0.05$ & 99.1 & 0.0800 & 5.618 & 5.780 & 0.5217 \\
\hline & 0.744 & $0.96 \pm 0.02$ & 97.3 & 0.0149 & 0.524 & 1.555 & 1.2203 \\
\hline \multirow[t]{2}{*}{$2^{\text {nd }}$ derivative } & 1.18 & $1.39 \pm 0.003$ & 97.9 & 0.0409 & 3.629 & 2.940 & 1.2370 \\
\hline & 0.93 & $1.16 \pm 0.01$ & 98.9 & 0 & 0 & 0 & 0 \\
\hline $3^{\text {rd }}$ derivative & 1.7478 & $1.96 \pm 0.003$ & 98.5 & 0.1914 & 3.826 & 9.770 & 1.2291 \\
\hline Milk(Buffalo) & - & & - & 0.1460 & 1.399 & 3.431 & 0.3530 \\
\hline $1^{\text {st }}$ derivative & - & 1.0 & - & 0.4075 & 5.090 & 10.91 & 0.8147 \\
\hline $2^{\text {nd }}$ derivative & - & 1.0071 & - & 0.5240 & 5.724 & 14.86 & 0.6577 \\
\hline $3^{\text {rd }}$ derivative & - & 1.0654 & - & 0.3088 & 2.602 & 8.281 & 0.6492 \\
\hline Milk(Cow) & - & & - & 0.1657 & 4.915 & 3.591 & 0.1469 \\
\hline $1^{\text {st }}$ derivative & - & 1.23 & - & 0.5835 & 6.559 & 13.55 & 0.0325 \\
\hline $2^{\text {nd }}$ derivative & - & & - & 0.9981 & 3.014 & 23.21 & 1.3550 \\
\hline $3^{\text {rd }}$ derivative & - & & - & .5265 & 6.229 & 11.95 & 0.5321 \\
\hline Milk(Mother) & - & & - & 0.0686 & 4.790 & 2.024 & 0.8205 \\
\hline $1^{\text {st }}$ derivative & - & 0.4297 & - & 0.0535 & 4.259 & 1.779 & 2.1004 \\
\hline $2^{\text {nd }}$ derivative & - & 0.4288 & - & 0.2672 & 2.942 & 8.901 & 1.9454 \\
\hline \multirow[t]{2}{*}{$3^{\text {rd }}$ derivative } & - & 0.5674 & - & 0.4747 & 6.256 & 11.95 & 0.3111 \\
\hline & & & & & & & \\
\hline Cabbage & - & 17.2 & - & 0.0779 & 1.267 & 2.158 & 1.1731 \\
\hline \multirow[t]{2}{*}{$1^{\text {st }}$ derivative } & - & 17.91 & - & 0.2465 & 4.524 & 6.552 & 1.4855 \\
\hline & 2.21 & $19.27 \pm 0.005$ & 99.27 & 0.2175 & 2.949 & 4.502 & 1.3681 \\
\hline \multirow[t]{2}{*}{$2^{\text {nd }}$ derivative } & - & 18.01 & - & 0.3781 & 2.196 & 9.994 & 0.6806 \\
\hline & 1.98 & $18.35 \pm 0.02$ & 95.7 & 0.3612 & 3.621 & 2.941 & 0.3992 \\
\hline \multirow[t]{2}{*}{$3^{\text {rd }}$ derivative } & - & 13.52 & - & 0.3644 & 0.891 & 12.83 & 1.3693 \\
\hline & 2.14 & $18.70 \pm 0.04$ & 96.7 & 0.3871 & 2.358 & 4.510 & 0.8414 \\
\hline $\begin{array}{c}\text { Vegetable } \\
\text { banana }\end{array}$ & - & 14.76 & - & 0.0642 & 0.843 & 2.074 & 1.6413 \\
\hline \multirow[t]{2}{*}{$1^{\text {st }}$ derivative } & - & 12.32 & - & 0.3348 & 2.565 & 11.59 & 0.3848 \\
\hline & 7.84 & $21.67 \pm 0.001$ & 95.9 & 0.3295 & 3.707 & 6.281 & 1.4971 \\
\hline \multirow[t]{2}{*}{$2^{\text {nd }}$ derivative } & - & 12.07 & - & 0.2805 & 6.287 & 3.664 & 0.0183 \\
\hline & 8.12 & $22.55 \pm 0.04$ & 98.6 & 0.4677 & 1.807 & 3.697 & 0.3617 \\
\hline \multirow[t]{2}{*}{$3^{\text {rd }}$ derivative } & - & 12 & - & 0.0735 & 2.793 & 2.915 & 0.6582 \\
\hline & 7.94 & $22.06 \pm 0.0003$ & 97.2 & 0.2782 & 4.126 & 3.780 & 1.0900 \\
\hline
\end{tabular}




\begin{tabular}{|c|c|c|c|c|c|c|c|}
\hline $\begin{array}{l}\text { Amruthapani } \\
\text { banana }\end{array}$ & - & 16.4 & - & 0.1254 & 6.477 & 3.633 & 0.2370 \\
\hline \multirow[t]{2}{*}{$1^{\text {st }}$ derivative } & - & 13.09 & - & 0.3098 & 2.175 & 11.26 & 1.037 \\
\hline & 3.79 & $19.68 \pm 0.0005$ & 97.5 & 0.1978 & 4.408 & 0.416 & 1.2293 \\
\hline \multirow[t]{2}{*}{$2^{\text {nd }}$ derivative } & - & 14.99 & - & 0.3502 & 5.014 & 11.11 & 1.358 \\
\hline & 4.22 & $20.31 \pm 0.01$ & 99.5 & 0.3309 & 1.745 & 0.260 & 1.120 \\
\hline \multirow[t]{2}{*}{$3^{\text {rd }}$ derivative } & - & 21.59 & - & 0.1388 & 3.193 & 3.0600 .7 & 1.155 \\
\hline & 3.86 & $19.22 \pm 0.002$ & 94.9 & 0.5060 & 2.534 & 12 & 0.5681 \\
\hline Tomato & - & 18.35 & - & 0.2239 & 6.918 & 5.782 & 1.7173 \\
\hline \multirow[t]{2}{*}{$1^{\text {st }}$ derivative } & - & 14.13 & - & 0.2068 & 4.220 & 6.967 & 0.6712 \\
\hline & 3.98 & $21.59 \pm 0.004$ & 96.7 & 0.3850 & 4.962 & 6.879 & 0.8615 \\
\hline \multirow[t]{2}{*}{$2^{\text {nd }}$ derivative } & - & 23.75 & - & 0.3709 & 4.837 & 7.433 & 2.2669 \\
\hline & 4.22 & $22.23 \pm 0.001$ & 98.5 & 0.1383 & 2.157 & 1.313 & 0.8894 \\
\hline \multirow[t]{2}{*}{$3^{\text {rd }}$ derivative } & - & 21.55 & - & 0.2805 & 1.951 & 6.197 & 0.1352 \\
\hline & 4.25 & $21.44 \pm 0.0002$ & 94.9 & 0.2302 & 3.398 & 3.250 & 0.3461 \\
\hline Wheat flour & - & 15.7 & - & 0.0506 & 3.143 & 1.532 & 1.681 \\
\hline \multirow{2}{*}{$1^{\text {st }}$ derivative } & - & 14.85 & - & 0.0835 & 2.792 & 2.677 & 1.0528 \\
\hline & 5.25 & $20.27 \pm 0.002$ & 96.8 & 0.0956 & 1.018 & 1.616 & 1.3032 \\
\hline \multirow[t]{2}{*}{$2^{\text {nd }}$ derivative } & - & 16.65 & - & 0.6531 & 2.398 & 18.68 & 1.7672 \\
\hline & 4.89 & $19.58 \pm 0.04$ & 94.7 & 0.0711 & 0.603 & 0.557 & 1.5477 \\
\hline \multirow[t]{2}{*}{$3^{\text {rd }}$ derivative } & - & 16.83 & - & 0.1115 & 2.947 & 3.154 & 1.3074 \\
\hline & 4.87 & $20.34 \pm 0.0005$ & 98.9 & 0.333 & 2.576 & 3.462 & 1.3218 \\
\hline Cucumber & - & 24.28 & - & 0.0268 & 1.495 & 0.526 & 2.2065 \\
\hline \multirow[t]{2}{*}{$1^{\text {st }}$ derivative } & - & 17.93 & - & 0.2942 & 5.742 & 7.809 & 0.8276 \\
\hline & 3.92 & $27.26 \pm 0.0019$ & 96.7 & 0.4363 & 2.619 & 13.83 & 0.6667 \\
\hline \multirow[t]{2}{*}{$2^{\text {nd }}$ derivative } & - & 23.21 & - & 0.17086 & 3.024 & 3.504 & 1.984 \\
\hline & 4.24 & $27.15 \pm 0.05$ & 95.2 & 0.0865 & 1.598 & 1.295 & 0.2449 \\
\hline \multirow[t]{2}{*}{$3^{\text {rd }}$ derivative } & - & 14.02 & - & 0.1527 & 5.010 & 5.138 & 0.9028 \\
\hline & 4.09 & $26.72 \pm 0.0006$ & 94.2 & 0.3989 & 1.488 & 3.416 & 0.3052 \\
\hline Rice(1010) & - & 14.5222 & - & 0.2017 & 0.772 & 3.968 & 2.2105 \\
\hline \multirow{2}{*}{$1^{\text {st }}$ derivative } & - & 8.4805 & - & 0.0595 & 4.471 & 2.004 & 1.445 \\
\hline & 5.00 & $13.280 \pm 0.001$ & 98.5 & 0.4499 & 3.666 & 7.695 & 0.6403 \\
\hline \multirow[t]{2}{*}{$2^{\text {nd }}$ derivative } & - & 13.7077 & - & 0.1770 & 1.810 & 3.689 & 0.8146 \\
\hline & 5.00 & $17.997 \pm 0.0003$ & 96.19 & 0.1118 & 1.962 & 2.098 & 0.5798 \\
\hline \multirow[t]{2}{*}{$3^{\text {rd }}$ derivative } & - & 11.808 & - & 0.2502 & 4.406 & 6.053 & 1.0907 \\
\hline & 5.00 & $16.00 \pm 0.02$ & 95.19 & 0.3112 & 0.141 & 6.183 & 0.9409 \\
\hline Rice(Masuria) & - & 21.68 & - & 0.1278 & 3.227 & 2.807 & 0.1855 \\
\hline \multirow[t]{2}{*}{$1^{\text {st }}$ derivative } & - & 17.4059 & - & 0.1078 & 1.161 & 2.948 & 1.3288 \\
\hline & 3.00 & $20.222 \pm 0.0001$ & 99.0 & 0.1547 & 1.859 & 3.282 & 0.8421 \\
\hline \multirow[t]{2}{*}{$2^{\text {nd }}$ derivative } & - & 19.589 & - & 0.2576 & 3.141 & 6.261 & 1.080 \\
\hline & 3.00 & $21.669 \pm 0.02$ & 95.9 & 03870 & 6.875 & 8.503 & 0.2279 \\
\hline \multirow[t]{2}{*}{$3^{\text {rd }}$ derivative } & - & 18.244 & - & 0.1483 & 2.601 & 3.870 & 1.0746 \\
\hline & 3.00 & $20.62 \pm 0.001$ & 97.0 & 0.2166 & 3.412 & 4.999 & 1.2175 \\
\hline Human Hair & $45.02^{33}$ & 41.2 & 91.5 & 0.0329 & 0.440 & 0.380 & 1.6724 \\
\hline $1^{\text {st }}$ derivative & & 45.73 & 101.5 & 0.0776 & 1.672 & 1.034 & 0.175 \\
\hline $2^{\text {nd }}$ derivative & & 44.59 & 99.0 & 0.0550 & 2.849 & 0.949 & 1.880 \\
\hline $3^{\text {rd }}$ derivative & & 45.59 & 101.2 & 0.0622 & 1.899 & 1.004 & 0.9556 \\
\hline
\end{tabular}


Table 6. Determination of copper in pharmaceutical preparations

\begin{tabular}{|c|c|c|c|c|c|c|c|c|}
\hline Pharmaceuticals & Form & 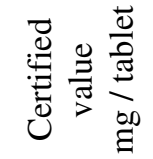 & 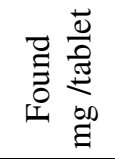 & 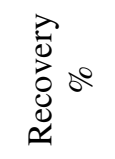 & 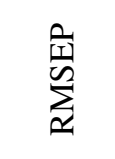 & $\frac{\text { Ty }}{\alpha}$ o & $\hat{\approx}$ & $\begin{array}{l}\overrightarrow{\tilde{n}} \\
\stackrel{\Delta}{1} \\
+\end{array}$ \\
\hline Supradyne & $\mathrm{CuSO}_{4}$. & 3.39 & 3.41 & 100.6 & 0.0459 & 3.2319 & 1.346 & 3.8511 \\
\hline $1^{\text {st }}$ derivative & $5 \mathrm{H}_{2} \mathrm{O}$ & & 3.57 & 105.3 & 0.0800 & 3.2740 & 2.2405 & 2.2925 \\
\hline $2^{\text {nd }}$ derivative & & & 3.78 & 111 & 0.4535 & 3.9085 & 10.910 & 0.6177 \\
\hline $3^{\text {rd }}$ derivative & & & 3.10 & 91.3 & 0.2079 & 2.7644 & 6.7150 & 2.1522 \\
\hline MULTIRICH & Copper & 50 & 53 & 106 & 0.4780 & 2.8202 & 9.684 & 2.4166 \\
\hline $1^{\text {st }}$ derivative & & & 48.86 & 97.72 & 0.3005 & 4.4118 & 7.873 & 1.1470 \\
\hline $2^{\text {nd }}$ derivative & & & 49.47 & 98.94 & 0.2348 & 2.3565 & 5.634 & 0.4754 \\
\hline $3^{\text {rd }}$ derivative & & & 52.07 & 104.14 & 0.3959 & 4.2369 & 10.032 & 1.5487 \\
\hline MULTIVITE & $\mathrm{CuSO}_{4}$. & 0.1 & 0.108 & 108 & 0.1420 & 0.0358 & 3.749 & 2.5074 \\
\hline $1^{\text {st }}$ derivative & $5 \mathrm{H}_{2} \mathrm{O}$ & & 0.092 & 92 & 0.1459 & 2.1845 & 4.531 & 1.3329 \\
\hline $2^{\text {nd }}$ derivative & & & 0.101 & 101 & 0.1642 & 3.0030 & 3.541 & 0.3624 \\
\hline $3^{\text {rd }}$ derivative & & & 0.105 & 105 & 0.3002 & 4.9610 & 7.4187 & 1.0059 \\
\hline GBION & Copper & 2.0 & 1.36 & 68.0 & 0.5284 & 0.0679 & 11.074 & 0.4895 \\
\hline $1^{\text {st }}$ derivative & & & 0.8623 & 43.1 & 0.1170 & 2.2943 & 3.8440 & 0.8891 \\
\hline $2^{\text {nd }}$ deri & & & 0.8922 & 44.6 & 0.2831 & 4.1110 & 9.0652 & 0.1854 \\
\hline $3^{\text {rd }}$ derivative & & & 0.954 & 47.7 & & 2.4457 & 2.6300 & 1.5883 \\
\hline NEXBLEND & $\mathrm{CuO}$ & 0.5 & 0.40 & 80 & 0.3149 & 4.5997 & 8.0009 & 1.8416 \\
\hline $1^{\text {st }}$ derivative & & $\mathrm{mg} / 15 \mathrm{~mL}$ & 0.3047 & 60.94 & 0.074 & 3.4494 & 2.4279 & 0.1666 \\
\hline $2^{\text {nd }}$ derivative & & & 0.3542 & 70.84 & 0.1780 & 2.1879 & 1.5519 & 4.6484 \\
\hline $3^{\text {rd }}$ derivative & & & 0.3148 & 62.96 & 0.0872 & 2.4457 & 2.6306 & 1.5883 \\
\hline
\end{tabular}

The infants not given breast milk, fed with buffalo's milk and cow's milk may have to been increased the bioavailability of copper and is associated with the acute phase reactions of number of diseased states, is always almost accompanied by hypercaeruloplasminaemia ${ }^{45}$. The content of copper determined in the human hair is $41.2-45.4 \mu \mathrm{g} / \mathrm{g}$. It is good coincidence with the values reported in the literature ${ }^{33}$.

The quantity of copper(II) in the common man dietaries like cabbage $(13.5-18 \mu \mathrm{g} / \mathrm{g})$, vegetable banana (12-14.7 $\mu \mathrm{g} / \mathrm{g})$, Amruthapani banana (13.1-21.5 $\mu \mathrm{g} / \mathrm{g})$, tomato (14.1-23.7 $\mu \mathrm{g} / \mathrm{g})$, wheat flour $(14.9-16.9 \mu \mathrm{g} / \mathrm{g})$, cucumber $(14-24.2 \mu \mathrm{g} / \mathrm{g})$, rice $1010(8.5-14.5 \mu \mathrm{g} / \mathrm{g})$, rice masuria (17.4-19.6 $\mu \mathrm{g} / \mathrm{g}$ ), were determined by this method, it was widely believed that most ostensibly healthy individuals consumed diets are to provide $2000 \mu \mathrm{g}$ of copper/day ${ }^{46}$. So the above diets are suggestive as good dietary for healthy individuals to supplement the require copper.

The estimation of copper in the pharmaceutical samples shows the efficiency of the method and sensitivity of the reagent than the methods reported in the literature ${ }^{33-38}$. However, in the case of GBion tablet determined value of $\mathrm{Cu}(\mathrm{II})$ is very low than the certified value, in all other cases the certified and the determined values are in good agreement hence, it was concluded the GBion tablets [Cotec health care pvt.ltd Uttaranchal. India.] Maintain substantial values than the certified.

The proposed spectrophotometric method is more selective. The standard addition method was used to determine $\mathrm{Cu}(\mathrm{II})$ in real samples, because of the incomplete release due to the interfering effects. The relative standard deviations representing the reproducibility 
and low detection limits in the determinations. The reagent used is highly specific. Hitherto no information in the literature used for the trace metal analysis. The proposed method shows the possibility of determination of ultra trace levels without the use of sophisticated instrumentation.

\section{Acknowledgement}

The authors are very thankful to Sri Anam Vivekananda Reddy Garu MLA Secretary and correspondent V. R. Colleges and High school committee, for providing facilities to carry out the proposed work and day to day inspiring encouragement.

\section{References}

1. Duffus J H, Toxicologia Ambiential, Omega, Barcelona, 1983.

2. Kosonen T, Uriu-Hare J Y, Clegg M S, Keen C L and Rucker R B, Bio Chem J., 1997, 327, 283.

3. Louma S N, Sci., Total Environ., 1983, 1, 28.

4. Kumar A, Hussain M F, Setake M and Puri D K, J Chin Chem Soc., 1984, 31, 55.

5. Lin J L and Cheng L F, J Chin Chem Soc., 1984, 31, 297.

6. Lin J L and Satake M, J Chin Chem Soc., 1985, 32, 105.

7. Chao M S and Chung C S, J Chin Chem Soc., 1989, 36, 301.

8. Patty F A, Industrial Hygiene and Toxicology, Inter Science Publishers New York, 1962, 2, 1033.

9. Shan Z F, Stud Trace Elements Health, 2006, 23(3), 66.

10. Cao H L, Stud Trace Elements Health, 2001, 18, 73.

11. Lau O W and Ho S Y, Anal Chim Acta, 1993, 280, 269-277.

12. Rigin V, Anal Chim Acta, 1993, 283, 895.

13. She Z and Wang Z, Fensi Huaxue., 1993, 21, 1313-1316.

14. Xie N, Huang C and Fu H D, Sepu., 1990, 8(2), 114.

15. She Z, Nie F and Chen Y, Fensi Huaxane., 1991, 19, 1272.

16. Qiao Z K, Hou Q Z and Lihua Jianyan, Hvaxue Fence, 1995, 31(2), 105.

17. Sun P P and Wu B C, Fenxi Shiyanshi, 1994, 13(5), 11.

18. Captain Gareia F, Captain - Vallvey L F, Gines - Fernandez D and Espinosa H P, Quim Anal., 1988, 7(4), 451.

19. Noroles A, Valladares L and Fresenius Z, Anal Chem., 1989, 334(1), 53-55.

20. Molina F, Fernandez G D, Bosque - Sendra M J B and Espinosa P, J Pharm Biomed Anal., 1988, 6(6-8), 1099.

21. Karayannis M I, Talanta, 1994, 41(10), 1645-1649.

22. Shinde V M and Khopkar S M, Anal Chem., 1969, 41(2), 342.

23. Terashima K and Tomioka H, Jap Anal., 1969, 18(8), 998.

24. Katiyar G S and Haldar B C, Indian J Chem Soc., 1984, 61(4), 353-355.

25. Ramanjaneyulu G, Ravendra Reddy P, Krishna Reddy V and Sreenivasulu Reddy T, The Open Anal Chem J., 2008, 2, 78-82.

26. Ghazy S E and Mostafa G A E, Egypt J Chem., 2002, 45, 855.

27. Campaigne E, Bosin T and Neiss E S, J Med Chem., 1967, 10, 270-271.

28. A.I Vogel, the text book of quantitative analysis Longman, Grout Pub London U.K, 1989.

29. Gofttschalk, Zeit Anal Chem., 1959, 167, 342.

30. Gomori G, Methods in Enzymology, 1955, 1, 141.

31. Ghazy S E, EI-Shazly R M, EI-Shahawi M S, AI-Hazmi G A A and EI.Asmy A A, $J$ Iranian Chem Soc., 2006, 3(2), 142-150. 
32. Rekha D, Suvardhan K, Suresh Kumar K, Reddyprasad P, Jayaraj B and Chiranjeevi P, J Serb Chem Soc., 2007, 72(3), 299-310.

33. Anant P Argekar and Ashok K Shetty, Anal Sci., 1996, 12, 255-258.

34. Mandalin Mathew and Narayana B, J Sci Ind Res., 2007, 66, 28-31.

35. Park C I, Kim H S and Cha K W, Bull Korean Chem Soc., 1999, 20(3), 352-354.

36. Qing-Zhouzhai, Bull Chem Soc Ethiop., 2009, 23(3), 327-335.

37. Ortha Turkoglu and Mostafa Soylak, J Chin Chem Soc., 2005, 52(3), 575-579.

38. Melisew Tadele Alula, Abdel-Maaboud I Mohamed and Adnan A Bekhit, Thai $J$ Pharm Sci., 2010, 34, 93-106.

39. Guidelines for drinking water quality $1993,2^{\text {nd }}$ Ed., v1.WHO Geneva.

40. Washington D C, National Academy of Science, 1977, 117.

41. Prasad P M N and Reddy Y V R, Electronic news: Letter on renuwal energy and environment, 2010, 7(1), 9-16.

42. Turnlund J R, Keyes W R, Anderson H L and Accord L L, Am J Clin Nutr., 1989, 49, 870-878.

43. Minor and Trace elements in breast milk 1989, WHO 9, Geneva.

44. Salmenpera.L, Perheentupa J and Siimes M A, Am J Clinical Nutr., 1986, 43, 251-257.

45. Under Wood E J, Trace Elements in Human and Animal Nutrition $4^{\text {th }}$ Edition Academic Press Newyork, 1977, 56-108.

46. National Research Council. Recommended Dietary Allowances $9^{\text {th }}$; Washington DC, National Academy of Science, 1980. 